OPEN ACCESS

Edited by:

Marco Cardinale,

Aspire Academy for Sports

Excellence, Qatar

Reviewed by:

Beat Knechtle,

University of Zurich, Switzerland Roger Enoka,

University of Colorado, USA

*Correspondence:

Daria Neyroud daria.neyroud@unil.ch

Specialty section

This article was submitted to

Exercise Physiology,

a section of the journal

Frontiers in Physiology

Received: 20 June 2016

Accepted: 15 August 2016

Published: 31 August 2016

Citation:

Neyroud D, Kayser B and Place N

(2016) Are There Critical Fatigue

Thresholds? Aggregated vs. Individual

Data. Front. Physiol. 7:376

doi: 10.3389/fphys.2016.00376

\section{Are There Critical Fatigue Thresholds? Aggregated vs. Individual Data}

\author{
Daria Neyroud ${ }^{1,2 *}$, Bengt Kayser ${ }^{1,2}$ and Nicolas Place ${ }^{1,2}$ \\ ${ }^{1}$ Institute of Sport Sciences, University of Lausanne, Lausanne, Switzerland, ${ }^{2}$ Department of Physiology, Faculty of Biology \\ and Medicine, University of Lausanne, Lausanne, Switzerland
}

The mechanisms underlying task failure from fatiguing physical efforts have been the focus of many studies without reaching consensus. An attractive but debated model explains effort termination with a critical peripheral fatigue threshold. Upon reaching this threshold, feedback from sensory afferents would trigger task disengagement from open-ended tasks or a reduction of exercise intensity of closed-ended tasks. Alternatively, the extant literature also appears compatible with a more global critical threshold of loss of maximal voluntary contraction force. Indeed, maximal voluntary contraction force loss from fatiguing exercise realized at a given intensity appears rather consistent between different studies. However, when looking at individual data, the similar maximal force losses observed between different tasks performed at similar intensities might just be an "artifact" of data aggregation. It would then seem possible that such a difference observed between individual and aggregated data also applies to other models previously proposed to explain task failure from fatiguing physical efforts. We therefore suggest that one should be cautious when trying to infer models that try to explain individual behavior from aggregated data.

Keywords: task failure, endurance performance, peak twitch, maximal voluntary contraction, critical threshold, neuromuscular fatigue

The typical answer when asking someone who just terminated a fatiguing task why stopped is "I just couldn't go on any further". If this suggests a conscious but forced decision to de-recruit the activated motor units for the task at hand, the underlying mechanisms forcing someone to terminate a fatiguing task are still not understood (Kayser, 2003). Given the great variety in physical tasks and their differing physiological constraints, it is unlikely that a single mechanism applies, just as it is likely that various types of effort will share some common pathways leading to the disengagement from the task at hand. For example, during dynamic exercise such as running or cycling, the cardiovascular strain is much greater than during an isometric contraction of a specific muscle group (Sidhu et al., 2013). For the former the sensation of effort is multimodal (e.g., breathlessness, palpitations, leg pain), while for the latter it is essentially related to the muscular effort per se (e.g., pain, loss of force) as the cardiorespiratory strain is limited.

The various mechanisms potentially implicated in exercise termination have been the focus of a great number of studies (for reviews see Kayser, 2003; Hunter et al., 2004; Marcora and Staiano, 2010; Enoka et al., 2011; Noakes, 2012; Amann et al., 2013; Enoka and Duchateau, 2016; Fan and Kayser, 2016; Taylor et al., 2016). In particular the role of peripheral fatigue, i.e., involving mechanisms located beyond the motor endplate, has been researched extensively. Functionally, 
peripheral fatigue can be evaluated by quantifying reductions in evoked forces induced by single or paired supramaximal electrical or magnetic stimulations delivered over a motor nerve trunk before and after a fatiguing exercise. If peripheral fatigue extent has been proposed as a key determinant causing task failure by some authors (Amann and Dempsey, 2008; Amann et al., 2009, 2013; Amann, 2011; Sidhu et al., 2014; Blain et al., 2016), its role in the termination of different types of muscular effort is still hotly debated (Marcora, 2009; Marcora and Staiano, 2010; Johnson et al., 2015; Morales-Alamo et al., 2015).

\section{A CRITICAL PERIPHERAL FATIGUE THRESHOLD AS A DETERMINANT OF TASK FAILURE?}

A recently postulated mechanism shared between various types of muscular effort is a so-called "critical peripheral fatigue threshold," proposed by Amann et al. $(2006,2009,2013)$ and Blain et al. (2016). According to those authors, peripheral fatigue normally does not exceed a certain individual critical threshold. If, during a given task, this threshold is reached, individuals either terminate it (open-ended tasks) or reduce the intensity (closedended tasks) (Amann et al., 2009). This critical peripheral fatigue threshold concept emerged from the observation of consistently reproducible peak twitch force reductions immediately following various cycling bouts to task failure (open-end) (Amann et al., 2006, 2007, 2009, 2011; Romer et al., 2007; Amann and Dempsey, 2008), as well as 5-km cycling time trials (closed-end) (Amann et al., 2006; Blain et al., 2016). Further support for such a critical peripheral fatigue threshold hypothesis was that at task failure of these open and closed-ended tasks, a greater reduction in peak twitch was reached following selective blockade of sensory afferents with intrathecal fentanyl injection compared to saline (Amann et al., 2009, 2011; Blain et al., 2016). Given the direct evidence of III-IV afferents involvement in exercise regulation from animal studies (Darques and Jammes, 1997; Dousset et al., 2004), this suggests that sensory type III-IV afferents might play a critical role in the regulation of a tightly regulated individual "permissible" extent of peripheral fatigue.

Yet, recent findings by Morales-Alamo et al. (2015) are difficult to reconcile with this notion of a critical fatigue threshold. In their hallmark study, the subjects performed 10 -s long all-out isokinetic sprints before, and 10 or $60 \mathrm{~s}$ after an incremental maximal cycling test. Immediately at task failure of the incremental exercise, a bilateral cuff was placed around both thighs and inflated to occlude leg blood flow and hence prevent metabolite clearance. Given the (measured) low levels of phosphocreatine (PCr) and increased levels of adenosine diphosphate (ADP) at task failure, mitochondrial respiration was still high when the cuffs were inflated and the authors calculated that the little oxygen remaining was depleted within the following $3 \mathrm{~s}$. Despite acidosis, anaerobic glycolysis and metabolites continued to accumulate during the ischemic recovery, as highlighted by the higher muscle lactate concentration at $60 \mathrm{~s}$ of occlusion compared to at $10 \mathrm{~s}$. The ischemic recovery thus induced a greater metabolic disturbance and, as such, it should be expected that type III and IV afferent firing was at the very least maintained if not increased during this period (Jankowski et al., 2013; Laurin et al., 2015). As the cuff was deflated only immediately before the beginning of the sprints performed either 10 or $60 \mathrm{~s}$ after the incremental maximal test, a poorer sprint performance was expected after the $60 \mathrm{~s}$ recovery period compared to the $10 \mathrm{~s}$ one. Surprisingly, not only was the power developed after $10 \mathrm{~s}$ higher than the maximum power reached at task failure of the preceding incremental exercise test, the sprint after $60 \mathrm{~s}$ of ischemic recovery reached higher power than the one after $10 \mathrm{~s}$. These findings suggest that despite a milieu interne expected to strongly stimulate type III-IV afferents, motor drive was not inhibited as much during the $10 \mathrm{~s}$ all-out sprints as compared to at task failure of the incremental test. Even though evoked force loss was not directly quantified in this study, nor were the experiments repeated after intrathecal fentanyl injection, the results nevertheless question a universal critical peripheral threshold hypothesis, at least for 10-s long all-out sprints.

Further evidence that questions the critical peripheral threshold comes from the different extents of evoked peak twitch reductions found at task failure from a cycling bout performed at $80 \%$ of peak power in different studies. Goodall et al. (2012) reported an average reduction of $20 \%$, Sidhu et al. (2014) of $46 \%$, and Amann et al. (2011) of 34\%, even though all three studies used an identical exercise task. One possible explanation for the lesser peak twitch decrease found at task failure by Goodall et al. (2012) than by Amann et al. (2011) could be that task failure can occur before reaching a critical peripheral fatigue. This possibility is supported by recent results obtained by Johnson et al. (2015), who reported that the extent of evoked force loss at task failure of a cycling bout was less when realized after prior upper body exercise. It is further possible that such differences between studies reporting reductions in evoked force might be explained by differences in the characteristics of the participants involved (e.g., different training status and other inter-individual variability). On the other hand, supposing that different participant cohorts were involved in each of their studies, it is striking how the different studies conducted by Amann and colleagues consistently found a reproducible peak twitch force loss. Nevertheless, given the above mentioned discrepancies, it would seem that the critical peripheral fatigue threshold hypothesis based on "interindividual consistency of the degree of end-exercise fatigue” (Broxterman et al., 2015) needs further testing.

To foster the debate we here review a series of recent studies in which losses in MVC and evoked forces as well as changes in voluntary activation level (VAL) induced by a fatiguing effort were quantified (Table 1). Studies were included if they involved exercise tasks where the subjects were asked to continue for as long as possible, i.e., until task failure, and quantified MVC and evoked force losses. The tasks varied from isometric contractions with various muscle groups to dynamic exercise tasks such as cycling at a fixed power output until task failure. There clearly are discrepancies in the extent of evoked force losses following a given exercise (see Table 1). However, attention should be paid to the time point at which evoked forces were evaluated in 
TABLE 1 | Summary of studies that quantified maximal voluntary contraction (MVC), evoked force and voluntary activation level (VAL) changes after various exercise.

\begin{tabular}{|c|c|c|c|c|c|c|c|}
\hline Study & Muscle & Fatiguing task & Post evaluation at & $\begin{array}{l}\text { TTF } \\
\mathbf{s}\end{array}$ & $\begin{array}{c}\text { MVC loss } \\
\%\end{array}$ & $\begin{array}{l}\text { Evoked force loss } \\
\qquad \%\end{array}$ & $\begin{array}{c}\text { VAL loss } \\
\%\end{array}$ \\
\hline Matkowski et al., 2011 & $\mathrm{KE}$ & $20 \%$ MVC to TF one leg & not specified & 295 & -37 & $-24^{a}$ & -13 \\
\hline Matkowski et al., 2011 & KE & $20 \%$ MVC to TF two legs & not specified & 245 & -26 & & -7 \\
\hline Neyroud et al., 2012 & $\mathrm{KE}$ & $20 \%$ MVC to TF & at TF & 246 & -51 & $-37^{a}$ & -7 \\
\hline Place et al., 2005 & $\mathrm{KE} 35^{\circ}$ & $20 \%$ MVC to TF & $20-30 s$ & 974 & -28 & $-3^{a}$ & -19 \\
\hline Place et al., 2005 & $\mathrm{KE} 75^{\circ}$ & $20 \%$ MVC to TF & $20-30 s$ & 398 & -28 & $-4^{\mathrm{a}}$ & -14 \\
\hline Place et al., 2007 & $\mathrm{KE}$ & $40 \%$ MVC to TF & $20-30 s$ & 101 & -16 & $-3^{\mathrm{a}} /-15^{\mathrm{b}}$ & -6 \\
\hline Kalmar and Cafarelli, 1999 & $\mathrm{KE}$ & $50 \%$ MVC to TF Pla & immediately after TF & 66 & -30 & $-55^{\mathrm{b}}$ & \\
\hline Kalmar and Cafarelli, 1999 & $\mathrm{KE}$ & $50 \%$ MVC to TF Caf & immediately after TF & 82.5 & -30 & $-55^{\mathrm{b}}$ & \\
\hline Neyroud et al., 2013 & KE & $50 \%$ MVC to TF & at TF & 77 & -34 & $-28^{a} /-36^{b}$ & -5 \\
\hline Amann et al., 2006 & $\mathrm{KE}$ & cycling at $83 \%$ peak power to TF in Nx & $2 \min 30$ & 489 & -9 & $-24^{\mathrm{a}} /-34^{\mathrm{b}}$ & \\
\hline Amann et al., 2006 & $\mathrm{KE}$ & cycling at $83 \%$ peak power to TF in $\mathrm{Hx}$ & $2 \min 30$ & 270 & -11 & $-23^{a} /-32^{b}$ & \\
\hline Amann et al., 2006 & $\mathrm{KE}$ & cycling at $83 \%$ peak power to TF in hyperoxia & $2 \min 30$ & 1162 & -9 & $-24^{\mathrm{a}} /-32^{\mathrm{b}}$ & \\
\hline Amann et al., 2011 & $\mathrm{KE}$ & cycling at $80 \%$ peak power to TF & $3 \mathrm{~min}$ & 522 & -10 & $-34^{b}$ & -1 \\
\hline Amann and Dempsey, 2008 & $\mathrm{KE}$ & cycling at $83 \%$ peak power to TF & $4 \min$ & $\sim 600$ & -10 & $-36^{b}$ & 0 \\
\hline Goodall et al., 2012 & $\mathrm{KE}$ & cycling at $80 \%$ peak power to TF in Nx & $2 \min 30$ & 486 & -17 & $-19^{b}$ & -9 \\
\hline Goodall et al., 2012 & $\mathrm{KE}$ & cycling at $80 \%$ peak power to TF in $\mathrm{Hx}$ & $2 \min 30$ & 216 & -25 & $-30^{b}$ & -18 \\
\hline 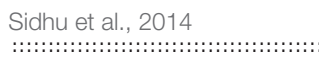 & $\mathrm{KE}$ & cycling at $80 \%$ peak power to TF & 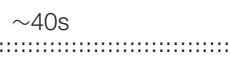 & 588 & -16 & $-46^{\mathrm{b}}$ & $\begin{array}{l}-10 \\
::::::::::\end{array}$ \\
\hline Rupp et al., 2015 & $\mathrm{KE}$ & $40 \%$ MVC to TF in Nx & immediately after TF & 458 & -18 & $-10^{a} /-18^{b}$ & -9 \\
\hline Rupp et al., 2015 & $\mathrm{KE}$ & $40 \%$ MVC to TF in $\mathrm{Hx}$ & immediately after TF & 449 & -16 & $-6^{a} /-16^{b}$ & -12 \\
\hline Neyroud et al., 2013 & PF & $50 \%$ MVC to TF & at TF & 220 & -30 & $-7^{a} /-1^{b}$ & -13 \\
\hline Yoon et al., 2007 & EF & $20 \%$ MVC to TF men & immediately after TF & 636 & -17 & $-23^{b}$ & -10 \\
\hline Yoon et al., 2007 & EF & $20 \%$ MVC to TF women & immediately after TF & 1020 & -32 & $-33^{b}$ & -17 \\
\hline Yoon et al., 2008 & EF & $20 \%$ MVC to TF young & immediately after TF & 864 & -27 & $-28^{b}$ & -14 \\
\hline Yoon et al., 2008 & $\mathrm{EF}$ & $20 \%$ MVC to TF old & immediately after TF & 1770 & -38 & $-33^{b}$ & -13 \\
\hline Neyroud et al., 2013 & $\mathrm{EF}$ & $50 \%$ MVC to TF & at TF & 72 & -40 & $-59^{\mathrm{a}} /-72^{\mathrm{b}}$ & -6 \\
\hline Yoon et al., 2007 & $\mathrm{EF}$ & $80 \%$ MVC to TF men & immediately after TF & 25 & -16 & $-37^{\mathrm{b}}$ & -4 \\
\hline Yoon et al., 2007 & EF & $80 \%$ MVC to TF women & immediately after TF & 24 & -15 & $-29^{b}$ & -6 \\
\hline Yoon et al., 2008 & $\mathrm{EF}$ & $80 \%$ MVC to TF young & immediately after TF & 24 & -15 & $-33^{b}$ & -4 \\
\hline $\begin{array}{l}\text { Yoon et al., } 2008 \\
::::::::::::::::::::::::::::::::::::::\end{array}$ & $\begin{array}{l}\mathrm{EF} \\
::::::::::::\end{array}$ & $\begin{array}{l}\text { 80\% MVC to TF old } \\
::::::::::::::::::::::::::::::::::::::::::::::::::::\end{array}$ & $\begin{array}{l}\text { immediately after TF } \\
::::::::::::::::::::::::::::: \text { : }\end{array}$ & $\begin{array}{l}32 \\
::::::::::\end{array}$ & $\begin{array}{c}-9 \\
::::::::::::\end{array}$ & $\begin{array}{c}-18^{\mathrm{b}} \\
:::::::::::::::::::::::::::\end{array}$ & -2 \\
\hline Fuglevand et al., 1993 & FDI & $20 \%$ MVC to TF & $\sim 30 \mathrm{~s}$ & 534 & -40 & $-55^{\mathrm{a}}$ & \\
\hline Fuglevand et al., 1993 & FDI & $35 \%$ MVC to TF & $\sim 30 \mathrm{~s}$ & 246 & -30 & $-54^{\mathrm{a}}$ & \\
\hline $\begin{array}{l}\text { Fuglevand et al., } 1993 \\
\text { :::::::::::::::::::::::::::::::::: }\end{array}$ & $\begin{array}{l}\mathrm{FDI} \\
:::::::::\end{array}$ & 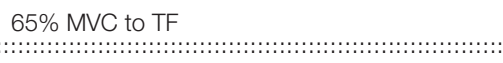 & $\begin{array}{l}\sim 30 \mathrm{~s} \\
::::::::::::::\end{array}$ & $\begin{array}{l}66 \\
:::::::\end{array}$ & $\begin{array}{c}-19 \\
:::::::::::::\end{array}$ & $-10^{\mathrm{a}}$ & $::::::::::::$ \\
\hline Neyroud et al., 2013 & ADD & $50 \% \mathrm{MVC}$ to TF & at TF & 114 & -37 & $-60^{\mathrm{a}} /-63^{\mathrm{b}}$ & -2 \\
\hline
\end{tabular}

TTF, time to task failure; TF, task failure; Nx, normoxia; Hx, hypoxia; KE, knee extensors; PF, plantar flexors; ADD, adductor pollicis; EF, elbow flexors and FDI, first dorsal interosseous. ${ }^{a}$ Indicates that peripheral fatigue extent was measured by evoking a $100-\mathrm{Hz}$ paired stimulation whereas ${ }^{b}$ means that it was measured by evoking a single stimulation.

these studies, as the delay between the moment of task failure and the subsequent evaluation affects the extent of the latter because of recovery (Neyroud et al., 2012; Froyd et al., 2013). As highlighted in Table 1, at task failure from a 20\% MVC sustained isometric contraction of the knee extensors, reductions in evoked forces varied from $3 \%$ in Place et al. (2005) to $24 \%$ in Matkowski et al. (2011) even though a similar break $(\sim 20 \mathrm{~s})$ was provided between task failure and the electrically-evoked contraction. Although single and paired stimuli are classically used as indexes of peripheral fatigue, it should be mentioned that the number of stimuli delivered might affect the factors constraining evoked force production (Parmiggiani and Stein, 1981). To sum up, in light of these studies, and given MVCs performed with similar levels of voluntary activation rates, the existence of a critical peripheral fatigue threshold does not appear obvious.

\section{COULD A MORE GLOBAL CRITICAL THRESHOLD BE INVOLVED IN TASK FAILURE?}

Alternatively, the extant literature on neuromuscular fatigue appears compatible with the existence of a more global critical threshold based on MVC force loss. Indeed, in view of the different studies reported in Table 1, it appears that at 
failure of tasks realized at a given intensity, MVC force losses were similar. A MVC force loss of $\sim 30-40 \%$ seems to be consistently observed following sustained isometric contractions performed with the knee extensors at 20\% MVC when the MVCs were realized a few seconds after task failure (Place et al., 2005; Matkowski et al., 2011). Similar MVC force losses were found when a similar task was performed with the elbow flexors (Yoon et al., 2007, 2008). This suggests that MVC force loss might be tightly regulated to not exceed a certain threshold.

Similarly to what was observed for evoked forces, the time at which MVC force loss is assessed affects its extent (Neyroud et al., 2012; Froyd et al., 2013). Yet, if results from studies evaluating MVC force loss at a similar time delay after task failure are compared, then its extent appears to be rather consistent. When sustained isometric contractions are performed, MVC force losses also appeared consistent between the various studies for a given intensity (c.f. Table 1). Interestingly, a similar MVC force loss was found following a 50\% MVC sustained isometric contraction performed to task failure with four different muscle groups, whereas reductions in evoked forces differed (Neyroud et al., 2013). Again, these results would support a tight regulation of MVC force loss. Accordingly, it can be hypothesized that instead of being limited by the extent of peripheral fatigue (reflected by reductions in evoked forces), exercise termination might result from a certain degree of
MVC force loss being reached, the latter being exercise-intensity dependent.

However, although this new hypothesis appears seductive, close examination of individual data shows that such a critical global threshold based on MVC force loss does not hold at the individual level and rather seems a mere artifact of data aggregation. For example, considering the data obtained by Place et al. (2005) following a sustained isometric contraction at $20 \%$ MVC to task failure performed with the knee extensors at two different knee angles ( $35^{\circ}$ vs. $75^{\circ}$ of knee flexion), it appeared that averaged MVC force losses were similar between the two tasks $\left(-28 \pm 16\right.$ and $-28 \pm 19 \%$ following the $35^{\circ}$ and $75^{\circ}$ task, respectively). Yet, when considering individual values, it can be seen that MVC force loss was greater following the $35^{\circ}$ task than the $75^{\circ}$ one in four of the nine participants, whereas the opposite was found in the five other participants (Figure 1A). It thus appears that the similar averaged MVC force loss observed in this study between the two different tasks was a mere reflection of half of the participants showing one behavior and the other half behaving in the opposite manner. A similar finding is observed from the results obtained in a study comparing MVC force loss induced by a sustained isometric contraction at 50\% MVC to task failure performed with four different muscle groups (Neyroud et al., 2013, Figure 1B).

The similar reduction in evoked forces reported between the two fatiguing tasks performed in Place et al. (2005) can also be
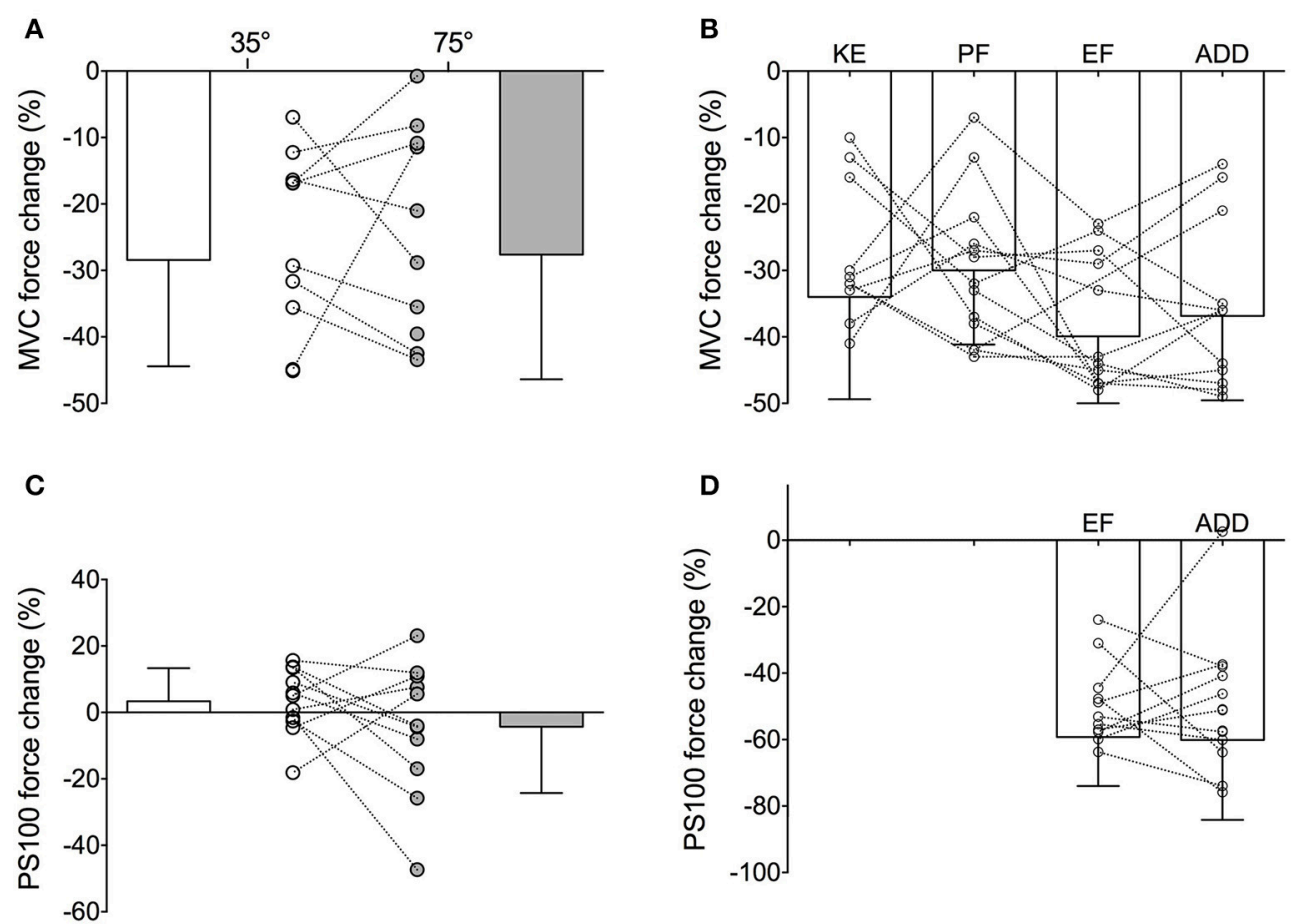

FIGURE 1 | Maximal voluntary contraction (MVC) force and 100-Hz evoked (PS100) force changes at task failure of (i) (A,C) a $20 \%$ MVC sustained isometric contraction performed with the knee extensors at knee angles of $35^{\circ}$ (unfilled) and $75^{\circ}$ (filled) of knee flexion and (ii) (B,D) a 50\% MVC sustained isometric contraction performed with four muscle groups. For the illustration of the present purpose, PS100 force loss was only represented for the elbow flexor and adductor pollicis tasks in panel D as peripheral fatigue extent differed in the two other muscle groups. KE, knee extensors; PF, plantar flexors; EF, elbow flexors and ADD, adductor pollicis. 
ascribed to cancelation of two opposite behaviors shown by the participants (some showing a greater evoked force reduction after the $35^{\circ}$ task whereas evoked forces were reduced to a greater in extent in some others after the $75^{\circ}$ task, Figure 1C). Similarly, in Neyroud et al. (2013), the evoked force loss measured at failure of the exercise involving elbow flexors and the adductor pollicis was similar, whereas a look at the individual values (Figure 1D) clearly showed that half the participants displayed greater reductions following the elbow flexor task and the other half following the adductor pollicis task.

As a critical threshold (global or peripheral) would be physiologically relevant only at the individual level, the above mentioned observations highlight the importance of considering interpretation of individual data and not only of group means, despite statistics. Indeed, when group means are compared and models/theories inferred from them (such as done when results of several studies are put together and new interpretations are

\section{REFERENCES}

Amann, M. (2011). Central and peripheral fatigue: interaction during cycling exercise in humans. Med. Sci. Sports Exerc. 43, 2039-2045. doi: 10.1249/MSS.0b013e31821f59ab

Amann, M., Blain, G. M., Proctor, L. T., Sebranek, J. J., Pegelow, D. F., and Dempsey, J. A. (2011). Implications of group III and IV muscle afferents for high-intensity endurance exercise performance in humans. J. Physiol. 589, 5299-5309. doi: 10.1113/jphysiol.2011.213769

Amann, M., and Dempsey, J. A. (2008). Locomotor muscle fatigue modifies central motor drive in healthy humans and imposes a limitation to exercise performance. J. Physiol. 586, 161-173. doi: 10.1113/jphysiol.2007. 141838

Amann, M., Eldridge, M. W., Lovering, A. T., Stickland, M. K., Pegelow, D. F., and Dempsey, J. A. (2006). Arterial oxygenation influences central motor output and exercise performance via effects on peripheral locomotor muscle fatigue in humans. J. Physiol. 575, 937-952. doi: 10.1113/jphysiol.2006. 113936

Amann, M., Proctor, L. T., Sebranek, J. J., Pegelow, D. F., and Dempsey, J. A. (2009). Opioid-mediated muscle afferents inhibit central motor drive and limit peripheral muscle fatigue development in humans. J. Physiol. 587, 271-283. doi: 10.1113/jphysiol.2008.163303

Amann, M., Romer, L. M., Subudhi, A. W., Pegelow, D. F., and Dempsey, J. A. (2007). Severity of arterial hypoxaemia affects the relative contributions of peripheral muscle fatigue to exercise performance in healthy humans. J. Physiol. 581, 389-403. doi: 10.1113/jphysiol.2007.129700

Amann, M., Venturelli, M., Ives, S. J., Mcdaniel, J., Layec, G., Rossman, M. J., et al. (2013). Peripheral fatigue limits endurance exercise via a sensory feedback-mediated reduction in spinal motoneuronal output. J. Appl. Physiol. 115, 355-364. doi: 10.1152/japplphysiol.00049.2013

Blain, G. M., Mangum, T. S., Sidhu, S. K., Weavil, J. C., Hureau, T. J., Jessop, J. E., et al. (2016). Group III/IV muscle afferents limit the intramuscular metabolic perturbation during whole body exercise in humans. J. Physiol. doi: 10.1113/jp272283. [Epub ahead of print].

Broxterman, R. M., Richardson, R. S., and Amann, M. (2015). Less peripheral fatigue after prior exercise is not evidence against the regulation of the critical peripheral fatigue threshold. J. Appl. Physiol. (1985) 119, 1520. doi: 10.1152/japplphysiol.00759.2015

Darques, J. L., and Jammes, Y. (1997). Fatigue-induced changes in group IV muscle afferent activity: differences between high- and low-frequency electrically induced fatigues. Brain Res. 750, 147-154. doi: 10.1016/S0006-8993(96) 01341-8

Dousset, E., Marqueste, T., Decherchi, P., Jammes, Y., and Grelot, L. (2004). Effects of neonatal capsaicin deafferentation on neuromuscular adjustments, inferred), caution should be taken to avoid drawing wrong conclusions by making ecological errors [i.e., deducing inferences about individual data from group averages (Sheppard, 2003)]. Models aiming to explain task failure and exercise performance should therefore be inferred from individual data and not averaged ones. However, for that to be possible, future studies should consider presenting both mean and individual data as proposed in Figure 1. Adopting such a manner of presenting results might lead to better comprehension of the mechanisms regulating exercise performance and responsible for task failure as both individual and mean data would be available to the reader.

\section{AUTHOR CONTRIBUTIONS}

All authors listed, have made substantial, direct and intellectual contribution to the work, and approved it for publication.

performance, and afferent activities from adult tibialis anterior muscle during exercise. J. Neurosci. Res. 76, 734-741. doi: 10.1002/jnr.20110

Enoka, R., Baudry, S., Rudroff, T., Farina, D., Klass, M., and Duchateau, J. (2011). Unraveling the neurophysiology of muscle fatigue. J. Electromyogr. Kinesiol. 21, 208-219. doi: 10.1016/j.jelekin.2010.10.006

Enoka, R., and Duchateau, J. (2016). Translating Fatigue to Human Performance. Med. Sci. Sports Exerc. doi: 10.1249/mss.0000000000000929. [Epub ahead of print].

Fan, J. L., and Kayser, B. (2016). Fatigue and Exhaustion in hypoxia: the role of cerebral oxygenation. High Alt. Med. Biol. 17, 72-84. doi: 10.1089/ ham.2016.0034

Froyd, C., Millet, G. Y., and Noakes, T. D. (2013). The development of peripheral fatigue and short-term recovery during self-paced high-intensity exercise. $J$. Physiol. 591, 1339-1346. doi: 10.1113/jphysiol.2012.245316

Fuglevand, A. J., Zackowski, K. M., Huey, K. A., and Enoka, R. M. (1993). Impairment of neuromuscular propagation during human fatiguing contractions at submaximal forces. J. Physiol. 460, 549-572. doi: 10.1113/ jphysiol.1993.sp019486

Goodall, S., Gonzalez-Alonso, J., Ali, L., Ross, E. Z., and Romer, L. M. (2012). Supraspinal fatigue after normoxic and hypoxic exercise in humans. J. Physiol. 590, 2767-2782. doi: 10.1113/jphysiol.2012.228890

Hunter, S. K., Duchateau, J., and Enoka, R. M. (2004). Muscle fatigue and the mechanisms of task failure. Exerc. Sport Sci. Rev. 32, 44-49. doi: 10.1097/00003677-200404000-00002

Jankowski, M. P., Rau, K. K., Ekmann, K. M., Anderson, C. E., and Koerber, H. R. (2013). Comprehensive phenotyping of group III and IV muscle afferents in mouse. J. Neurophysiol. 109, 2374-2381. doi: 10.1152/jn.01067.2012

Johnson, M. A., Sharpe, G. R., Williams, N. C., and Hannah, R. (2015). Locomotor muscle fatigue is not critically regulated after prior upper body exercise. J. Appl. Physiol. (1985) 119, 840-850. doi: 10.1152/japplphysiol.00072.2015

Kalmar, J. M., and Cafarelli, E. (1999). Effects of caffeine on neuromuscular function. J. Appl. Physiol. 87, 801-808.

Kayser, B. (2003). Exercise starts and ends in the brain. Eur. J. Appl. Physiol. 90, 411-419. doi: 10.1007/s00421-003-0902-7

Laurin, J., Pertici, V., Dousset, E., Marqueste, T., and Decherchi, P. (2015). Group III and IV muscle afferents: role on central motor drive and clinical implications. Neuroscience 290, 543-551. doi: 10.1016/j.neuroscience. 2015.01.065

Marcora, S. (2009). Perception of effort during exercise is independent of afferent feedback from skeletal muscles, heart, and lungs. J. Appl. Physiol. 106, 2060-2062. doi: 10.1152/japplphysiol.90378.2008

Marcora, S. M., and Staiano, W. (2010). The limit to exercise tolerance in humans: mind over muscle? Eur. J. Appl. Physiol. 109, 763-770. doi: 10.1007/s00421-0101418-6 
Matkowski, B., Place, N., Martin, A., and Lepers, R. (2011). Neuromuscular fatigue differs following unilateral vs. bilateral sustained submaximal contractions. Scand. J. Med. Sci. Sports 21, 268-276. doi: 10.1111/j.1600-0838.2009.01040.x

Morales-Alamo, D., Losa-Reyna, J., Torres-Peralta, R., Martin-Rincon, M., PerezValera, M., Curtelin, D., et al. (2015). What limits performance during whole body incremental exercise to exhaustion in humans? J. Physiol. 593, 4631-4648. doi: $10.1113 /$ JP270487

Neyroud, D., Maffiuletti, N. A., Kayser, B., and Place, N. (2012). Mechanisms of fatigue and task failure induced by sustained submaximal contractions. Med. Sci. Sports Exerc. 44, 1243-1251. doi: 10.1249/MSS.0b013e318245cc4d

Neyroud, D., Ruttimann, J., Mannion, A. F., Millet, G. Y., Maffiuletti, N. A., Kayser, B., et al. (2013). Comparison of neuromuscular adjustments associated with sustained isometric contractions of four different muscle groups. J. Appl. Physiol. 114, 1426-1434. doi: 10.1152/japplphysiol.01539.2012

Noakes, T. D. (2012). Fatigue is a brain-derived emotion that regulates the exercise behavior to ensure the protection of whole body homeostasis. Front. Physiol. 3:82. doi: $10.3389 /$ fphys.2012.00082

Parmiggiani, F., and Stein, R. B. (1981). Nonlinear summation of contractions in cat muscles. II. Later facilitation and stiffness changes. J. Gen. Physiol. 78, 295-311. doi: 10.1085/jgp.78.3.295

Place, N., Maffiuletti, N. A., Ballay, Y., and Lepers, R. (2005). Twitch potentiation is greater after a fatiguing submaximal isometric contraction performed at short vs. long quadriceps muscle length. J. Appl. Physiol. 98, 429-436. doi: 10.1152/japplphysiol.00664.2004

Place, N., Martin, A., Ballay, Y., and Lepers, R. (2007). Neuromuscular fatigue differs with biofeedback type when performing a submaximal contraction. J. Electromyogr. Kinesiol. 17, 253-263. doi: 10.1016/j.jelekin.2006. 04.001

Romer, L., Haverkamp, H. C., Amann, M., Lovering, A. T., Pegelow, D. F., and Dempsey, J. A. (2007). Effect of acute severe hypoxia on peripheral fatigue and endurance capacity in healthy humans. Am. J. Physiol. Regul. Integr. Comp. Physiol. 292, 598-606. doi: 10.1152/ajpregu.00269.2006

Rupp, T., Racinais, S., Bringard, A., Lapole, T., and Perrey, S. (2015). Modulation of exercise-induced spinal loop properties in response to oxygen availability. Eur. J. Appl. Physiol. 115, 471-482. doi: 10.1007/s00421-0143032-5

Sheppard, L. (2003). Insights on bias and information in group-level studies. Biostatistics 4, 265-278. doi: 10.1093/biostatistics/4.2.265

Sidhu, S. K., Cresswell, A. G., and Carroll, T. J. (2013). Corticospinal responses to sustained locomotor exercises: moving beyond single-joint studies of central fatigue. Sports Med. 43, 437-449. doi: 10.1007/s40279-0130020-6

Sidhu, S. K., Weavil, J. C., Venturelli, M., Garten, R. S., Rossman, M. J., Richardson, R. S., et al. (2014). Spinal mu-opioid receptor-sensitive lower limb muscle afferents determine corticospinal responsiveness and promote central fatigue in upper limb muscle. J. Physiol. 592, 5011-5024. doi: 10.1113/jphysiol.2014.275438

Taylor, J. L., Amann, M., Duchateau, J., Meeusen, R., and Rice, C. L. (2016). Neural contributions to muscle fatigue: from the brain to the muscle and back again. Med. Sci. Sports Exerc. doi: 10.1249/mss.0000000000000923. [Epub ahead of print].

Yoon, T., De-Lap, B. S., Griffith, E. E., and Hunter, S. K. (2008). Age-related muscle fatigue after a low-force fatiguing contraction is explained by central fatigue. Muscle Nerve 37, 457-466. doi: 10.1002/mus.20969

Yoon, T., Schlinder Delap, B., Griffith, E. E., and Hunter, S. K. (2007). Mechanisms of fatigue differ after low- and high-force fatiguing contractions in men and women. Muscle Nerve 36, 515-524. doi: 10.1002/mus.20844

Conflict of Interest Statement: The authors declare that the research was conducted in the absence of any commercial or financial relationships that could be construed as a potential conflict of interest.

Copyright (c) 2016 Neyroud, Kayser and Place. This is an open-access article distributed under the terms of the Creative Commons Attribution License (CC BY). The use, distribution or reproduction in other forums is permitted, provided the original author(s) or licensor are credited and that the original publication in this journal is cited, in accordance with accepted academic practice. No use, distribution or reproduction is permitted which does not comply with these terms. 